\title{
Adaptación al español del instrumento sobre cultura organizacional de Denison ${ }^{1}$
}

\section{Spanish adaptation of the "Denison Organizational Culture Survey"}

\author{
Tomás Bonavia ${ }^{2 *}$, Vicente J. Prado ${ }^{1 *}$, Alejandra García-Hernández ${ }^{3 * *}$ \\ *Universidad de Valencia \\ Facultad de Psicología, Departamento de Psicología Social, España \\ **Ingenio (CSIC-UPV) \\ Institute for Innovation and Knowledge Management \\ Ciudad Politécnica de la Innovación, España
}

(Recepción: Febrero 2010 - Aceptación: Julio 2010)

\begin{abstract}
Resumen
En este estudio se presentan los resultados de la adaptación a grupos I+D universitarios de habla hispana de la encuesta sobre cultura organizacional de Denison. Este cuestionario, compuesto por 60 ítems que identifican 12 tipos culturales, que a su vez se agrupan en cuatro dimensiones, ha demostrado su influencia para aumentar la efectividad organizacional. La adaptación al castellano que aquí se propone ha sido aplicada a 488 personas pertenecientes a 39 grupos de I+D tecnológicos de la Universidad Politécnica de Valencia. Los resultados obtenidos muestran unas propiedades psicométricas adecuadas para la adaptación realizada en cuanto a validez y fiabilidad, que replican casi en su totalidad la estructura del modelo de la versión original.
\end{abstract}

Palabras claves: adaptación cuestionario, fiabilidad, validez, análisis factorial confirmatorio.

\begin{abstract}
This article presents a Spanish adaptation of Denison Organizational Culture Survey. This questionnaire consists of 60 items, grouped in 12 sub-scales, which identify 4 cultural dimensions. These dimensions have been widely study and their influence in organizational effectiveness is accepted. The Spanish adaptation proposed here has been applied in 488 participants of $39 \mathrm{I}+\mathrm{D}$ technological groups of Polytechnic University of Valencia. Results show adequate psychometric properties, so we can conclude that the Spanish adaptation almost fully replicates the structure of the original version in English.
\end{abstract}

Key words: adaptation measure/survey, reliability, validity, confirmatory factor analysis.

1 Agradecimientos: Este trabajo se enmarca dentro de un proyecto de investigación aprobado por el Ministerio de Educación y Ciencia titulado: "Influencia de la cultura organizativa y el flujo de conocimiento sobre el rendimiento científico de los grupos de I+D tecnológicos. Aplicación en la Universidad Politécnica de Valencia” (SEJ2005_08603).

2 Correspondencia a: Tomás Bonavia y Vicente J. Prado. Av. Blasco Ibáñez, 21, 46010 VALENCIA (España, Spain). E-mail: tomas.bonavia@uv.es, vicente.prado@uv.es.

3 Correspondencia a: Alejandra García Hernández. Camino de Vera s/n, Edificio 8E, 46022 VALENCIA (España, Spain). E-mail: algarhe1@upvnet.upv.es. 


\section{Adaptación al español del instrumento sobre cultura organizacional de Denison}

De entre todos los instrumentos en habla inglesa dedicados al estudio de la cultura organizacional, hemos optado por adaptar el cuestionario propuesto por Denison y colaboradores, denominado Denison Organizational Culture Survey (Denison y Neale, 1994, 2000). El cuestionario (a partir de ahora DOCS) es una encuesta estandarizada desarrollada para la medición y evaluación de la cultura organizativa. Es un instrumento autoadministrado de sencilla y rápida aplicación y de fácil comprensión. Consta de 60 ítems, 5 para a cada una de las 12 subescalas o índices propuestos en el modelo de cultura organizacional de Denison. Este modelo agrupa las 12 subescalas en 4 dimensiones o rasgos culturales (lo que hace un total de 15 ítems y 3 subescalas por dimensión). Cada ítem está codificado en una escala tipo Likert de cinco puntos (desde $1=$ completamente en desacuerdo hasta $5=$ completamente de acuerdo). La validez y fiabilidad del instrumento se han demostrado satisfactorias (Denison, Janovics y Young, 2005; Denison, Janovics, Young y Cho, 2006). El instrumento plantea, además, una representación gráfica de los resultados que hacen muy atractiva su interpretación. Otra ventaja indiscutible de esta encuesta es que la comunidad científica dispone de la versión completa de la misma, que puede ser utilizada sin fines comerciales con permiso del autor, no estando sujeto su uso a rígidas limitaciones como ocurre con otros cuestionarios sobre cultura.

Este instrumento y su modelo teórico de base han sido aplicados a una gran cantidad de organizaciones públicas y privadas (más de 700 según sus autores) de diferentes países. Son muy abundantes las publicaciones sobre el mismo, tanto teóricas como aplicadas. Incluyendo a modo de muestra, solamente aquéllas en las que aparece el autor principal, podemos encontrar las siguientes: Denison (1990, 1996, 2001), Denison y Mishra (1995), Denison y Neale (1994), Denison y Spreitzer (1991), Denison, Haaland y Goeltzer (2003), Denison, Hart y Kahn (1996), Denison, Lief y Ward (2004), Fey y Denison (2003) y, Smerek y Denison (2007).

El instrumento fue construido tras una revisión profusa de la investigación acerca de cómo la cultura influye en la efectividad organizacional (Denison, 1990, 2001). Esta finalidad explícita que determina claramente su objetivo lo diferencia de la mayoría de los demás cuestionarios sobre cultura organizativa, lo que supone otro importante valor añadido. Profundiza en el análisis a nivel de los valores organizativos atendiendo a la clásica distinción de Schein (1985). Estos valores se agrupan en cuatro rasgos culturales que han demostrado una fuerte influencia sobre la efectividad que alcanzan las organizaciones y que son: implicación, consistencia, adaptabilidad y misión (como veremos más adelante).

Es posible encontrar en la literatura científica otros instrumentos para evaluar la cultura organizacional, la mayoría provenientes del mundo anglosajón. Para una revisión de estos instrumentos pueden consultarse, entre otros: Price y Mueller (1986) que revisan cerca de 300 medidas de diferentes constructos organizacionales incluida la cultura; Ashkanasy, Broadfoot y Falkus (2000) que hallan 18 escalas publicadas sólo en el periodo que oscila entre 1975 y 1992; la revisión realizada por Scott, Mannion, Davies y Marshall (2003) sobre herramientas para la evaluación de la cultura organizativa y el clima, en especial referidas a los hospitales y ambientes médicos; o la realizada por Kimberly y Cook (2008) también sobre diferentes constructos organizacionales e instrumentos de medición.

Algunos de estos instrumentos en inglés se han traducido al castellano: "The Kilman-Saxton Culture-Gap Survey" (Kilman y Saxton, 1984) en Rodríguez (2005); "Organizational Culture Inventory" (Cooke y Lafferty 1987), el cual se puede conseguir contactando con la consultora Human Synergistics que dispone de los derechos sobre el mismo; o "The FOCUS Questionnaire" (Van Muijen et al., 1999), el cual fue elaborado ya en su origen en varios idiomas. Que sepamos, ninguno de estos cuestionarios ha sido analizado mediante análisis factorial confirmatorio con muestras españolas. Éste será, precisamente, uno de los objetivos que nos proponemos llevar a cabo con el DOCS en este artículo.

Existe, por tanto, un desfase importante en la disponibilidad de instrumentos válidos sobre cultura organizacional adaptados al castellano, en comparación a lo que sucede en otras zonas del mundo. Es verdad que se ha hecho un esfuerzo por desarrollar instrumentos propios en nuestro 
idioma (algunos ejemplos son: Bonavia y Quintanilla, 1996; Marcone y Martín, 2003; Martínez, 1994; Reyes y Zambrano, 1991; Sánchez y Alonso, 1997; Topa, Morales y Palací, 2005), pero su uso dificulta la comparación de los resultados obtenidos en nuestro país con los estudios que se publican en las revistas más prestigiosas. Nuestra investigación pretende ser una contribución más en este contexto que ayude a reducir estas diferencias, al tiempo que amplia a otros públicos el uso idiomático de un cuestionario sobre cultura organizacional que ha demostrado su utilidad e interés tanto teórico como aplicado.

\section{Método}

\section{Participantes}

Este estudio forma parte de un proyecto de investigación más amplio que pretende relacionar la cultura organizacional en los grupos de I+D universitarios con la gestión del conocimiento y la efectividad de estos grupos. Por este motivo, como primer paso, nos proponemos la adaptación y validación del DOCS como instrumento para evaluar la cultura organizacional. La adaptación española para grupos de I+D universitarios se administró a 488 personas pertenecientes a 39 grupos de $\mathrm{I}+\mathrm{D}, 17$ de ciencias básicas y 22 de ciencias técnicas de la Universidad Politécnica de Valencia (UPV). Para la elección de los grupos se recurrió a la información contenida en la base de datos de la UPV conocida como Sénia. Sénia es un sistema informático para la gestión de la actividad investigadora que se integra con otros servicios de información personalizada que ofrece la UPV (bases de datos de recursos humanos, CTT, alumnado, etc.).

La participación media de los integrantes de los diferentes grupos ha sido en torno al 68,35\%, con un mínimo de un $12,5 \%$ y un máximo del $100 \%$. De los 488 encuestados 260 son Personal Docente e Investigador (PDI), 111 Becarios de investigación y 52 son PAS (Personal Auxiliar y de Servicios). Se desconoce la categoría laboral de los 65 restantes. Por otro lado, en lo que respecta a la posesión o no del grado de Doctor, 183 de los encuestados afirman ser doctores mientras que 115 dicen no serlo (190 no han indicado su condición). No se recogieron datos relativos a la edad o el sexo de los encuestados para no poner gravemente en peligro su anonimato, lo que hubiera posiblemente impedido la realización de esta investigación.

\section{Instrumento}

Más arriba ya se han presentado algunos datos del Denison Organizational Culture Survey (DOCS). En lo que respecta a la estructura del cuestionario se compone de 12 subescalas o índices que se agrupan en 4 dimensiones o rasgos culturales (Denison, 2001). Veámoslo con más detalle siguiendo las palabras de su autor.

\section{Implicación (Involvement)}

Las organizaciones efectivas dan poder a sus miembros, se organizan en equipos y desarrollan las capacidades humanas a todos los niveles. Los miembros de estas organizaciones están comprometidos con su trabajo y sienten que son una parte importante de la organización. Las personas de cualquier nivel jerárquico participan de las decisiones que afectarán su trabajo y perciben que éste está directamente relacionado con los objetivos de la organización. En el cuestionario, estos aspectos están medidos por las tres subescalas siguientes:

- Empowerment (Empoderamiento, a diferencia de lo que haremos con el resto de vocablos, preferimos mantener aquí la denominación en inglés, ya que son varias las traducciones del mismo que se han propuesto pero ninguna ha sido plenamente aceptada): Los individuos tienen autoridad, iniciativa y capacidad para dirigir su propio trabajo. Esto genera un sentimiento de pertenencia y responsabilidad hacia la organización (ítems 1 al 5).

- Trabajo en equipo (Team Orientation): El valor reside en el trabajo cooperativo hacia objetivos comunes, sintiéndose todos los empleados responsables del mismo. La organización depende de los esfuerzos del trabajo en equipo para alcanzar los objetivos (ítems 6 al 10). 
- Desarrollo de capacidades (Capability Development): La organización invierte de forma continuada en el desarrollo de las competencias y habilidades de sus empleados con el objeto de mantener su competitividad y aprovechar nuevas oportunidades de negocio (ítems 11 al 15).

\section{Consistencia (Consistency)}

La investigación ha mostrado que las organizaciones son efectivas cuando son consistentes y están bien integradas. El comportamiento de las personas está marcado por un conjunto de valores que induce a líderes y seguidores a alcanzar acuerdos (aun cuando pueda existir divergencia en las opiniones), de manera que las actividades de la organización queden coordinadas adecuadamente. Las organizaciones con estas características tienen una cultura fuerte y distintiva que influye firmemente en el comportamiento de sus trabajadores. La consistencia es una potente fuente de estabilidad e integración interna resultado de una visión compartida y un alto grado de conformidad. Este rasgo cultural es medido a través de tres índices que son:

- Valores centrales (Core Values): Los miembros de la organización comparten una serie de valores que crean un sentimiento de identidad y un conjunto claro de expectativas (ítems 16 al 20).

- Acuerdo (Agreement): Los miembros de la organización son capaces de lograr acuerdos en los temas esenciales. Esto implica, por un lado, un nivel de acuerdo tácito, y por otro, la capacidad de reconciliar diferencias cuando ocurren (ítems 21 al 25).

- Coordinación e integración (Coordination and Integration): Diferentes áreas y unidades de la organización son capaces de trabajar conjuntamente para alcanzar objetivos comunes. Las fronteras organizativas no suponen barreras para desarrollar un buen trabajo (items 26 al 30).

\section{Adaptabilidad (Adaptability)}

Las organizaciones excesivamente integradas ofrecen a menudo dificultades para cambiar y adaptarse a nuevos entornos. Integración interna y adaptación externa pueden ser difíciles de compatibilizar, pero es necesario para lograr organizaciones efectivas. Las organizaciones adaptativas toman a sus clientes como guía, asumen riesgos y aprenden de sus errores, y tienen capacidad y experiencia en introducir cambios. Continuamente están mejorando la capacidad de crear valor para sus clientes. Las organizaciones con elevada adaptabilidad usualmente experimentan crecimiento en las ventas e incremento de las cuotas de mercado. Está dimensión cultural se mide por medio de tres subescalas:

- Orientación al cambio (Creating Change): La organización está capacitada para crear nuevos espacios conducentes al cambio. Es capaz de interpretar adecuadamente el entorno del negocio, reaccionar rápidamente a las modas vigentes y anticipar futuros cambios (ítems 31 al 35).

- Orientación al cliente (Customer Focus): La organización conoce a sus clientes y anticipa sus futuras necesidades. Refleja el grado en el que la organización está guiada por la preocupación de satisfacer a sus clientes (ítems 36 al 40).

- Aprendizaje organizativo (Organizational Learning): La organización recibe, interpreta y transforma señales del entorno en oportunidades que alienten la innovación, ganando conocimiento y desarrollando capacidades (ítems 41 al 45).

\section{Misión (Mission)}

Quizás el rasgo cultural más importante de todos sea el sentimiento de misión. Las organizaciones que no saben a dónde se dirigen, usualmente acaban en algún lugar no previsto. Las organizaciones 
exitosas tienen un propósito y una dirección precisa que define las metas organizacionales y los objetivos estratégicos, al tiempo que cuentan con una visión clara de cómo será la organización en el futuro. Las organizaciones con más problemas son a menudo aquéllas que han de cambiar su misión principal. Se requiere un fuerte liderazgo que defina una visión de futuro y construya una cultura que apoye dicha visión. Los tres índices que componen esta dimensión son:

- Dirección y propósitos estratégicos (Strategic Direction and Intention): Claras intenciones estratégicas expresan el propósito de la organización y muestran el modo en como todos los empleados pueden contribuir al desarrollo de la organización (ítems 46 al 50).

- Metas y objetivos (Goals and Objectives): A la misión, la visión y la estrategia pueden vincularse las metas y objetivos que faciliten a cada empleado una dirección precisa a su trabajo (ítems 51 al 55).

- Visión (Vision): La organización tiene una imagen compartida sobre la manera en que desea ser reconocida en el futuro. Esta perspectiva común encarna los valores nucleares y captura los corazones y las mentes de las personas que componen la empresa, proporcionando guía y dirección (ítems 56 al 60).

\section{Proceso de adaptación}

Para la versión castellana del DOCS se siguieron los estándares metodológicos internacionales recomendados por la International Test Comision (ITC) para una adaptación correcta de un instrumento de un contexto idiomático a otro (Hambleton, 1994; Hambleton, 1996; Muñiz y Hambleton, 2000). En concreto, se siguieron los pasos que se resumen a continuación:

1) El primero consistió en asegurarse de la existencia en el contexto e idioma español de los diferentes constructos utilizados en el cuestionario original. Aunque con algunas salvedades, se reconoció en líneas generales esta realidad. Además, dado el interés existente en el ámbito científico de realizar estudios transculturales, se consideró que emplear una adaptación de dicho cuestionario podía ser más interesante que crear uno nuevo.

2) Tras ponernos en contacto con Daniel Denison, quien mostró una buena disposición a participar en esta investigación, recibimos su versión en castellano del cuestionario original. Hubo que revisarla a fondo ya que necesitaba diferentes retoques idiomáticos y contextuales: en el caso de algunos ítems, se hizo necesario también adaptarlos al ámbito concreto de la investigación científica (lo que debería tenerse en cuenta de cara a la posterior utilización de la versión que ahora se publica). El proceso que se siguió en esta fase fue el siguiente. Cada miembro del equipo de investigación realizó una traducción de los ítems al castellano. Dichas traducciones fueron debatidas en diversas sesiones de trabajo hasta que finalmente se llegó a un consenso, teniendo en cuenta también para ello la versión proporcionada por Denison. Aunque todos los miembros del equipo son de nacionalidad española, un par de ellos residieron una parte considerable de su vida en EEUU. Asimismo, uno de los investigadores que participó en este proceso cuenta con una reconocida experiencia en traducción de inglés al castellano y viceversa.

3) Una vez se dispuso de una versión española del cuestionario adaptada al ámbito de la investigación científica, se realizó una traducción del castellano al inglés por un profesional cualificado y con experiencia al que se instruyó en algunos de los conceptos básicos que se utilizaban en el citado cuestionario. Seguidamente se procedió a revisar la versión original y la retrotraducida y se realizaron las correcciones necesarias en la versión española.

4) Posteriormente, se llevó a cabo un estudio piloto con el instrumento adaptado aplicándolo presencialmente a una muestra compuesta por 22 personas de 2 grupos de investigación pertenecientes al Instituto de la Gestión de la Innovación y del Conocimiento (INGENIO). Una vez se hubo cumplimentado, se les entrevistó para conocer su opinión sobre el cuestionario, la comprensión de las instrucciones, la redacción de los ítems, etc., así como las posibles sugerencias o cambios a considerar. Como consecuencia de ello, se introdujeron algunas modificaciones en la versión castellana del instrumento. 
5) Finalmente, se procedió a la aplicación empírica de esta última versión a una muestra de 488 personas, entre investigadores, becarios y personal técnico. Tras el análisis de los resultados psicométricos de los que se informan en este trabajo, se llegó a la versión final del instrumento que ahora se presenta.

\section{Procedimiento}

El procedimiento empleado para la obtención de la información se compuso de cinco fases diferentes. En la primera fase, tras identificar a los responsables de los grupos de I+D de la Universidad Politécnica de Valencia se procedió a contactar con los mismos, ya fuera mediante correo electrónico, en persona y/o telefónicamente (generalmente se empleó más de un canal para aumentar la participación). Una vez realizado ese primer contacto, como segundo paso se remitió a los responsables un ejemplar del cuestionario completo, entre los que se encontraba la adaptación del DOCS, y una carta informándoles de las características del estudio y animándoles a participar.

A continuación, en el momento en que se obtuvo su consentimiento para formar parte de esta investigación, se inició la tercera fase, en la que los responsables indicaron el tamaño y composición actual de sus grupos (PAS, PDI y Becarios). A partir de esta información, se les suministró los cuestionarios requeridos y la carta de presentación junto con sus respectivos sobres, más una urna o buzón donde poder depositarlos una vez fueran cumplimentados, todo ello para garantizar el anonimato y la confidencialidad de las respuestas obtenidas. En la penúltima fase el responsable del grupo y/u otra persona del mismo (promotor interno) se encargó de repartirlos y de animar nuevamente a la participación. Pasadas dos semanas aproximadamente se recogió la urna. Tras recabar la información se preparó un informe preliminar con los resultados del grupo y su comparación con el resto de grupos encuestados (quinta fase).

La duración de este proceso en los diferentes grupos varió entre uno y cuatro meses, ya que en varias ocasiones se requirió cierta insistencia tanto para iniciar el pase de cuestionarios, como para aumentar la representatividad del grupo en cuanto al volumen de participantes. Con el fin de asegurar la confidencialidad y la imposibilidad de identificar a miembros concretos dentro de cada grupo, no se incluyeron aspectos demográficos como la edad, el género, etc., habituales en este tipo de estudios.

\section{Plan de análisis}

El análisis estadístico de los datos se realizó mediante el paquete estadístico SPSS (versión 15.0) y el programa EQS 6.1 ambos para Windows. En primer lugar se calcularon los estadísticos descriptivos más importantes para cada ítem. También se calcularon las correlaciones entre las variables evaluadas, tanto entre las 12 subescalas como entre las 4 dimensiones principales. A continuación se llevaron a cabo los análisis de consistencia interna. Tras estos cálculos preliminares se procedió al estudio de la estructura factorial del instrumento, para lo cual se realizaron diferentes análisis factoriales exploratorios, empleando en todos ellos el método de máxima verosimilitud con rotación varimax (que fue el que utilizó en su día el autor para validar el cuestionario original), más cinco análisis factoriales confirmatorios (AFC).

Por otra parte, en lo que respecta a los AFC, dada la complejidad del modelo por la gran cantidad de variables que se debían incluir en el mismo (60 variables -ítems- que se agrupan en 12 subescalas, que a su vez se agrupan en 4 dimensiones o factores de segundo orden) se decidió optar por dos estrategias paralelas aunque complementarias. Primero, se puso a prueba el modelo para cada una de las 4 dimensiones a nivel de ítem (Implicación, Consistencia, Adaptabilidad y Misión). Cada modelo presentaba tres subescalas que correlacionaban entre sí y que estaban formadas por 5 ítems o variables cada una. Segundo, se planteó un modelo más general que consideró las relaciones entre las 4 dimensiones culturales y las 12 subescalas (a razón de 3 subescalas por dimensión). 


\section{Resultados}

\section{Análisis de ítems}

Se realizó un análisis de los 60 ítems que componen la adaptación del DOCS. En la Tabla 1 se presenta la redacción final de los ítems y, para cada ítem, su media, la desviación típica la correlación ítem-total, y el alpha de Cronbach si se elimina el elemento. Los ítems 15, 24, 29, 34, 39, 43, 50 y 58 (marcados en la tabla por un asterisco) evalúan de forma inversa el constructo al que se refieren, por lo que fueron recodificados previamente.

Tabla 1: Media, desviación típica (d.t.), correlación ítem-total (rjx) y alfa de Cronbach si se elimina el elemento $(\alpha .-\mathrm{x})$

\begin{tabular}{|c|c|c|c|c|c|}
\hline ITEMS & $\mathbf{N}$ & Media & d.t & rjx & $\alpha_{\cdot}-\mathbf{x}$ \\
\hline $\begin{array}{l}\text { 1. La mayoría de los miembros de este grupo están muy } \\
\text { comprometidos con su trabajo. }\end{array}$ & 486 & 4,28 &, 752 & ,526 & ,967 \\
\hline $\begin{array}{l}\text { 2. Las decisiones con frecuencia se toman en el nivel que } \\
\text { dispone de la mejor información. }\end{array}$ & 484 & 3,87 & ,955 &, 589 & ,967 \\
\hline $\begin{array}{l}\text { 3. La información se comparte ampliamente y se puede } \\
\text { conseguir la información que se necesita. }\end{array}$ & 487 & 3,83 & 1,086 & ,610 & ,967 \\
\hline $\begin{array}{l}\text { 4. Cada miembro cree que puede tener un impacto positi- } \\
\text { vo en el grupo. }\end{array}$ & 487 & 3,88 &, 859 &, 552 & ,967 \\
\hline $\begin{array}{l}\text { 5. La planificación de nuestro trabajo es continua e impli- } \\
\text { ca a todo el mundo en algún grado. }\end{array}$ & 487 & 3,47 & 1,103 & ,670 & ,966 \\
\hline $\begin{array}{l}\text { 6. Se fomenta activamente la cooperación entre los dife- } \\
\text { rentes grupos de esta organización. }\end{array}$ & 486 & 3,23 & 1,120 &, 582 & ,967 \\
\hline $\begin{array}{l}\text { 7. Trabajar en este grupo es como formar parte de un } \\
\text { equipo. }\end{array}$ & 487 & 3,90 & 1,085 & ,665 & ,966 \\
\hline $\begin{array}{l}\text { 8. Acostumbramos a realizar las tareas en equipo, en vez } \\
\text { de descargar el peso en la dirección. }\end{array}$ & 486 & 375 & ,954 & ,470 & ,967 \\
\hline $\begin{array}{l}\text { 9. Los grupos y no los individuos son los principales } \\
\text { pilares de esta organización. }\end{array}$ & 483 & 3,31 & 1,112 & ,488 & ,967 \\
\hline $\begin{array}{l}\text { 10. El trabajo se organiza de modo cada persona entiende } \\
\text { la relación entre su trabajo y los objetivos de la organiza- } \\
\text { ción. }\end{array}$ & 484 & 3,38 & 1,023 &, 716 & ,966 \\
\hline $\begin{array}{l}\text { 11. La autoridad se delega de modo que las personas } \\
\text { puedan actuar por sí mismas. }\end{array}$ & 487 & 3,74 & 1,017 &, 554 & ,967 \\
\hline $\begin{array}{l}\text { 12. Las capacidades del "banquillo" (los futuros líderes } \\
\text { en el grupo) se mejoran constantemente. }\end{array}$ & 485 & 3,42 & ,973 & ,605 & ,967 \\
\hline $\begin{array}{l}\text { 13. Este grupo invierte continuamente en el desarrollo de } \\
\text { las capacidades de sus miembros. }\end{array}$ & 487 & 3,63 & 1,090 & ,638 & ,966 \\
\hline $\begin{array}{l}\text { 14. La capacidad de las personas es vista como una fuente } \\
\text { importante de ventaja competitiva. }\end{array}$ & 485 & 3,84 & 1,030 & ,607 & ,967 \\
\hline $\begin{array}{l}\text { 15. A menudo surgen problemas porque no disponemos } \\
\text { de las habilidades necesarias para hacer el trabajo. * }\end{array}$ & 487 & 3,54 & 1,069 & ,284 & ,968 \\
\hline 16. Los líderes y directores practican lo que pregonan. & 485 & 3,68 & 1,078 &, 718 & ,966 \\
\hline $\begin{array}{l}\text { 17. Existe un estilo de dirección característico con un } \\
\text { conjunto de prácticas distintivas. }\end{array}$ & 483 & 3,56 & ,908 & ,483 & ,967 \\
\hline
\end{tabular}


18. Existe un conjunto de valores claro y consistente que rige la forma en que nos conducimos.

19. Ignorar los valores esenciales de este grupo te ocasionará problemas.

20. Existe un código ético que guía nuestro comportamiento y nos ayuda a distinguir lo correcto.

21. Cuando existen desacuerdos, trabajamos intensamente para encontrar soluciones donde todos ganen.

22. Este grupo tiene una cultura "fuerte".

23. Nos resulta fácil lograr el consenso, aun en temas difíciles.

24. A menudo tenemos dificultades para alcanzar acuerdos en temas clave.*

25. Existe un claro acuerdo acerca de la forma correcta e incorrecta de hacer las cosas.

26. Nuestra manera de trabajar es consistente y predecible.

27. Las personas de diferentes grupos de esta organización tienen una perspectiva común.

28. Es sencillo coordinar proyectos entre los diferentes grupos de esta organización.

29. Trabajar con alguien de otro grupo de esta organización es como trabajar con alguien de otra organización. * 30. Existe une buena alineación de objetivos entre los diferentes niveles jerárquicos.

31. La forma que tenemos de hacer las cosas es flexible y fácil de cambiar.

32. Respondemos bien a los cambios del entorno.

33. Adoptamos de continuo nuevas y mejores formas de hacer las cosas.

34. Los intentos de realizar cambios suelen encontrar resistencias.*

35. Los diferentes grupos de esta organización cooperan a menudo para introducir cambios.

36. Los comentarios y recomendaciones de nuestros clientes conducen a menudo a introducir cambios.

37. La información sobre nuestros clientes influye en nuestras decisiones.

38. Todos tenemos una comprensión profunda de los deseos y necesidades de nuestro entorno.

39. Nuestras decisiones ignoran con frecuencia los intereses de los clientes.*

40. Fomentamos el contacto directo de nuestra gente con los clientes

41. Consideramos el fracaso como una oportunidad para aprender y mejorar.

42. Tomar riesgos e innovar son fomentados y recompensados.

43. Muchas ideas "se pierden por el camino".*

44. El aprendizaje es un objetivo importante en nuestro trabajo cotidiano.

\begin{tabular}{|c|c|c|c|c|}
\hline 87 & 3,62 & ,968 & ,736 & ,96 \\
\hline 482 & 3,27 & ,993 &,- 010 & 0 \\
\hline 85 & 3,41 & 1,030 & ,596 & 9 \\
\hline 35 & 3,52 & 1,015 & ,650 & 9 \\
\hline 35 & 3,70 & ,966 & ,612 & \\
\hline 36 & 3,55 & 995 &, 550 & \\
\hline 35 & 3,55 & 994 & ,465 & 9 \\
\hline 36 & 3,53 & 969 & ,660 & 9 \\
\hline 37 & 3,65 & 883 & ,561 & 9 \\
\hline 86 & 3,11 & 1,073 & 606 & 9 \\
\hline 86 & 3,05 & 1,050 & ,572 & 9 \\
\hline 32 & 3,31 & 1,098 &, 316 & 9 \\
\hline 483 & 3,36 & ,932 & ,675 & ,9 \\
\hline 85 & 3,45 & ,927 & ,499 & ,9 \\
\hline 484 & 3,64 & ,828 & ,590 & ,9 \\
\hline 85 & 3,59 & ,917 & ,641 & ,96 \\
\hline 483 & 3,23 & 978 & ,428 & 9 \\
\hline 483 & 2,98 & ,939 &, 524 &, 9 \\
\hline 77 & 3,24 & 871 & ,446 & ,9 \\
\hline 77 & 3,48 & ,866 & ,332 & ,9 \\
\hline 84 & 3,28 & ,976 & 614 & ,9 \\
\hline 7 & 3,60 & 940 & ,469 & 9 \\
\hline 75 & 3,26 & ,933 & ,442 & ,9 \\
\hline 483 & 3,72 & 875 & ,447 & 9 \\
\hline 483 & 3,36 & 987 & ,598 & \\
\hline 483 & 3,00 & 1,076 & ,488 & \\
\hline A & 4,17 & ,888 & ,581 & \\
\hline
\end{tabular}


45. Nos aseguramos que "la mano derecha sepa lo que está haciendo la izquierda".

46. Esta organización tiene un proyecto y una orientación a largo plazo.

47. Nuestra estrategia sirve de ejemplo a otras organizaciones.

48. Esta organización tiene una misión clara que le otorga sentido y rumbo a nuestro trabajo.

49. Esta organización tiene una clara estrategia de cara al futuro.

50. La orientación estratégica de esta organización no me resulta clara.*

51. Existe un amplio acuerdo sobre las metas a conseguir.

52. Los líderes y directores fijan metas ambiciosas pero realistas.

53. La Dirección nos conduce hacia los objetivos que tratamos de alcanzar.

54. Comparamos continuamente nuestro progreso con los objetivos fijados.

55. Las personas de esta organización comprenden lo que hay que hacer para tener éxito a largo plazo.

56. Tenemos una visión compartida de cómo será esta organización en el futuro.

57. Los líderes y directores tienen una perspectiva a largo plazo.

58. El pensamiento a corto plazo compromete a menudo nuestra visión a largo plazo.*

59. Nuestra visión genera entusiasmo y motivación entre nosotros.

60. Podemos satisfacer las demandas a corto plazo sin comprometer nuestra visión a largo plazo.

$\begin{array}{lllll}484 & 3,39 & 1,076 & , 608 & , 967 \\ 483 & 3,78 & 1,034 & , 670 & , 966 \\ 478 & 3,24 & , 974 & , 558 & , 967 \\ 484 & 3,53 & 1,026 & , 761 & , 966 \\ 485 & 3,51 & 1,030 & , 705 & , 966 \\ 484 & 3,33 & 1,186 & , 544 & , 967 \\ 485 & 3,39 & , 944 & , 684 & , 966 \\ 484 & 3,63 & , 977 & , 680 & , 966 \\ 484 & 3,59 & , 966 & , 772 & , 966 \\ 484 & 3,48 & , 966 & , 619 & , 967 \\ 484 & 3,57 & , 968 & , 577 & , 967 \\ 484 & 3,06 & , 975 & , 692 & , 966 \\ 483 & 3,75 & , 933 & , 621 & , 967 \\ 482 & 3,03 & 1,000 & , 336 & , 967 \\ 483 & 3,45 & , 995 & , 709 & , 966 \\ 480 & 3,40 & , 881 & , 580 & , 967 \\ & & & & \\ 48\end{array}$

* Ítems inversos

En general todos los ítems parecen contribuir adecuadamente al conjunto de la escala, es decir, presentan una correlación relativamente alta con el total del cuestionario (la correlación media de los diferentes ítems que componen el cuestionario con la escala global es 0,57 ). No obstante se observa un ítem que presenta una correlación muy baja con el conjunto de la escala, se trata del ítem 19 "Ignorar los valores esenciales de este grupo te ocasionará problemas", cuyo índice $(-0,01)$ nos indica que apenas existe relación entre este ítem y los restantes, además de que esta casi nula relación lo es en sentido contrario al esperado. Si se eliminara este ítem, la consistencia interna de la subescala a la que pertenece mejoraría sustancialmente. Este ítem corresponde originariamente a la subescala Empowerment que a su vez pertenece a la dimensión más general denominada Implicación. Salvo esta cuestión, tras comparar los valores observados en la muestra española con los obtenidos en la muestra original (Denison, Janovics y Young, 2005; Denison, Janovics, Young y Cho, 2006) constatamos, en general, que los valores son muy similares.

\section{Correlaciones}

También se calcularon los coeficientes de correlación de Pearson entre las 12 subescalas y entre las 4 dimensiones. Las correlaciones entre todas las subescalas resultaron positivas, estadísticamente significativas al nivel de 0,01 y en general relativamente altas (casi la totalidad de las correlaciones 
se encuentran entre 0,50 y 0,76 ). La correlación más baja (aunque significativa) se encuentra entre Orientación al cliente y Visión con un valor de 0,39. En lo que respecta a las 4 dimensiones, se observan correlaciones muy altas (todas ellas superiores a 0,70 ) y significativas al nivel de 0,01 .

Si comparamos los valores obtenidos por los autores en su muestra original (Denison, Janovics y Young, 2005; Denison, Janovics, Young y Cho, 2006) con los valores obtenidos en la muestra española observaremos que, de nuevo, no existen casi diferencias. Las diferencias más altas se observan en la correlación entre Visión y Coordinación e integración (dif.= 0,13 ) seguida por Visión y Orientación al cambio (dif.=0,11). En ambas, la correlación entre dichos índices era superior en el cuestionario original. En el resto de comparaciones, apenas se encuentran diferencias siendo éstas menores a 0,01 en la mayoría de los casos.

\section{Análisis de fiabilidad}

La fiabilidad del instrumento, tanto del cuestionario en general como de las diferentes dimensiones y subescalas que lo componen, fue examinada calculando su consistencia interna mediante el índice alpha de Cronbach. Toda esa información junto a los valores obtenidos en la escala original se recogen en la Tabla 2.

Los valores encontrados muestran, en general, una adecuada consistencia interna para el cuestionario en la muestra española, superior incluso a la mostrada por la escala original en algunos casos. El alpha de Cronbach del cuestionario en su conjunto es de 0,97 y para las diferentes dimensiones y subescalas que lo componen se encuentra por encima de 0,70 . La única subescala que presenta puntuaciones inferiores a 0,70 es Valores centrales $(\alpha=0,68)$, que mejoraría hasta 0,78 si se elimina el ítem 19, que ya ha mostrado ciertos problemas como se ha comentado con anterioridad. En general, se puede afirmar que los valores obtenidos son adecuados y muy similares a los que encontraron los autores del cuestionario original en su muestra (Denison, Janovics y Young, 2005; Denison, Janovics, Young y Cho, 2006).

Tabla 2: Comparación de la fiabilidad del cuestionario original (Denison, Janovics y Young, 2005; Denison, Janovics, Young y Cho, 2006) y de su adaptación al castellano

\begin{tabular}{lcc}
\hline & $\begin{array}{c}\alpha \text { Cronbach } \\
\text { (Adaptación) }\end{array}$ & $\begin{array}{c}\alpha \text { Cronbach } \\
(\text { Original) }\end{array}$ \\
\hline Implicación & $\mathbf{0 , 9 0}$ & $\mathbf{0 , 9 0}$ \\
Empowerment & 0,79 & 0,77 \\
Trabajo en equipo & 0,80 & 0,83 \\
Desarrollo de capacidades & 0,72 & 0,70 \\
Consistencia & $\mathbf{0 , 8 7}$ & $\mathbf{0 , 8 8}$ \\
Valores centrales & 0,68 & 0,70 \\
Acuerdo & 0,80 & 0,75 \\
Coordinación e integración & 0,70 & 0,78 \\
Adaptabilidad & $\mathbf{0 , 8 7}$ & $\mathbf{0 , 8 7}$ \\
Orientación al cambio & 0,78 & 0,76 \\
Orientación al cliente & 0,78 & 0,73 \\
Aprendizaje organizativo & 0,70 & 0,75 \\
Misión & $\mathbf{0 , 9 3}$ & $\mathbf{0 , 9 2}$ \\
Dirección y propósitos estratégicos & 0,88 & 0,85 \\
Metas y Objetivos & 0,86 & 0,80 \\
Visión & 0,76 & 0,78 \\
\hline
\end{tabular}




\section{Análisis factorial exploratorio}

El siguiente paso que se llevó a cabo dentro del procedimiento de adaptación del DOCS, fue analizar su validez de contenido o de constructo. Con el objetivo de contrastar el modelo subyacente en el cuestionario, es decir, la entidad y rigor de los constructos medidos dentro de un marco teórico coherente (Muñiz, 1998), se han realizado análisis factoriales tanto de tipo exploratorio como confirmatorios.

En los diferentes análisis factoriales exploratorios realizados, el método de extracción escogido fue el de máxima verosimilitud con rotación varimax, puesto que ese fue el método empleado por los autores en la validación del cuestionario original. En el primero, se tomaron los 60 ítems como variables a evaluar sin limitar el número de factores, introduciendo como restricción que los autovalores fueran superiores a 1. En el siguiente análisis factorial exploratorio se redujeron los factores a 12, y en el penúltimo análisis a 4 factores. El último análisis consistió en extraer los factores de segundo orden (las cuatro dimensiones culturales) a partir de las 12 subescalas. Los resultados obtenidos en ellos sugieren que el modelo planteado por los autores de la versión original del cuestionario es, al menos parcialmente, admisible en la muestra española, ya que hemos observado que se replica la estructura de gran parte del cuestionario, aunque con diferencias, tanto en lo que respecta a sus subescalas como a sus dimensiones (los investigadores que lo deseen, pueden disponer de estos análisis solicitándolos a los autores de este artículo). Por ello, en este punto, se decidió recurrir a una serie de AFC para replicar el modelo teórico y los constructos latentes.

\section{Análisis factorial confirmatorio}

Para realizar los cinco AFC se utilizó el programa EQS 6.1 para Windows. Para la estimación de la bondad de ajuste de los diferentes modelos propuestos se empleó la estimación por máxima verosimilitud, y se aplicaron los test de Wald y de Lagrange para observar las sugerencias en cuanto a la inclusión o eliminación de algunas de las relaciones entre las variables consideradas en cada modelo. Aunque son muchos los índices que cabe utilizar para medir el grado de ajuste no existe un consenso claro sobre su uso. Por ello, en el presente artículo, nos centraremos en algunos de los más comunes como los propuestos por Maccallum y Austin (2000): Non-normed fit index (NNFI), Comparative fit index (CFI), Goodness of fit index (GFI), Ajusted goodness of fit index (AGFI); siendo habitual considerar un buen ajuste cuando los valores en estos índices son cercanos a 1,00 (Byrne, 1994). El último índice que utilizaremos en este artículo y que ha sido usado profusamente en la literatura científica es el Standardized root mean-square residual (SRMR), que sugiere un buen ajuste del modelo cuando su valor es inferior a 0,05 (Byrne, 1994). En la Tabla 3 se resumen los principales indicadores que hemos obtenido en los diferentes modelos y que comentaremos a continuación.

Tabla 3: Índices de bondad de ajuste de los diferentes modelos planteados

\begin{tabular}{lccccccc}
\hline \multicolumn{1}{c}{ MODELO } & $\chi 2$ & $\chi 2 / \mathrm{Df}$ & NNFI & CFI & GFI & AGFI & SRMR \\
\hline 1. Compromiso & 235,112 & 2,702 & 0,934 & 0,946 & 0,940 & 0,917 & 0,046 \\
2. Consistencia & 421,148 & 4,841 & 0,843 & 0,870 & 0,885 & 0,841 & 0,062 \\
3. Adaptabilidad & 309,575 & 3,558 & 0,881 & 0,901 & 0,918 & 0,887 & 0,059 \\
4. Misión & 369,867 & 4,251 & 0,915 & 0,930 & 0,900 & 0,862 & 0,042 \\
5. Factores de segun- & 174,899 & 3,644 & 0,940 & 0,956 & 0,920 & 0,870 & 0,034 \\
do orden & & & & & &
\end{tabular}




\section{MODELO 1: Replicación empírica de la dimensión Implicación}

En este primer modelo, se analizó si los primeros quince ítems del cuestionario se agrupan en 3 factores (o subescalas): Empowerment (ítems 1 al 5), Trabajo en equipo (ítems 6 al 10) y Desarrollo de capacidades (ítems 11 al 15). Además se indicó que dichos factores estaban correlacionados entre sí. Dados los valores relativamente bajos de los residuos estandarizados o error promedio $(0,0348)$ y el error promedio de los elementos fuera de la diagonal $(0,0398)$, parece que el modelo en cuestión presenta un buen ajuste. No obstante, dichos resultados deben ser contrastados con la distribución de los residuos estandarizados o distribución de los errores promedio. En lo que respecta a la distribución de los errores, siguen una distribución más o menos simétrica y relativamente centrada en 0 (nótese en este punto que el intervalo entre 0,1 y $-0,1$ recoge en torno al $95 \%$ de los errores). Por último, si observamos las iteraciones que ha tenido que realizar el modelo hasta conseguir converger parece que, si bien es cierto que ha requerido 8 iteraciones, también lo es que a partir de la cuarta el cambio medio en las estimaciones de los parámetros del modelo no ha sufrido apenas modificaciones y se ha estabilizado en valores bajos, lo que según Byrne (1994) puede significar que nos encontramos ante un ajuste adecuado. Además, los resultados de los diferentes índices analizados sugieren que el modelo es adecuado ya que son superiores a .90 y el SRMR inferior a 0,05 .

En definitiva, estos análisis parecen justificar la estructura interna de la adaptación del cuestionario por lo que se refiere a las subescalas que componen la dimensión Implicación. Además, los test de Wald y de Lagrange no sugieren que el modelo mejore al eliminar ninguna de las relaciones entre las variables planteadas. Así, parece que se confirma la hipótesis de que los primeros 15 ítems del cuestionario pertenecen a una misma dimensión o rasgo cultural, que a su vez se divide en 3 subescalas (Empowerment, Trabajo en equipo y Desarrollo de capacidades) formadas cada una de ellas por cinco ítems.

\section{MODELO 2: Replicación empírica de la dimensión Consistencia}

En este modelo, los siguientes 15 ítems del cuestionario se deben agrupar en 3 factores: Valores centrales (ítems 16 al 20), Acuerdo (ítems 21 al 25) y Coordinación e integración (ítems 26 al 30). También se indicó que dichos factores estaban correlacionados entre sí. Este modelo presenta valores superiores al anterior en lo que respecta a los residuos estandarizados o error promedio $(0,0449)$ y en lo referente al error promedio de los elementos fuera de la diagonal $(0,0513)$. Además los valores extremos también son superiores $(0,250)$. No obstante, dichos resultados deben ser contrastados con la distribución de los residuos estandarizados o distribución de los errores promedio. En lo que respecta a la distribución de los errores, siguen una distribución más o menos simétrica y relativamente centrada en 0 (nótese en este punto que el intervalo entre 0,1 y $-0,1$ recoge en torno al $89,16 \%$ de los errores residuales, cuando lo ideal sería una cifra cercana al 100\%). Por último, si observamos las iteraciones que ha tenido que realizar el modelo hasta converger, se han requerido 8 iteraciones y no ha sido antes de la sexta que el cambio medio en las estimaciones de los parámetros del modelo no ha sufrido modificaciones y se ha estabilizado en valores bajos.

Los resultados de los diferentes índices de bondad del modelo sugieren un ajuste no demasiado bueno, puesto que los valores obtenidos son inferiores a 0,90 aunque cercanos al límite, mientras que el valor de SRMR es ligeramente superior a 0,05. En definitiva, la adaptación del DOCS, al menos en lo que se refiere a las subescalas que componen la dimensión Consistencia (Valores centrales, Acuerdo y Coordinación e integración, formada cada una de ellas por cinco ítems), no parece replicarse totalmente en la muestra española. No obstante, dado que los valores se encuentran relativamente cercanos a lo que se considera adecuado, tampoco podemos determinar que esta estructura interna sea completamente incorrecta. El modelo mejora si se elimina el ítem 19, ítem que ya ha demostrado ser problemático en otros apartados anteriores. De hecho, atendiendo al test de Wald, el modelo mejora sensiblemente si se elimina la relación entre el ítem 19 y la subescala de Valores centrales. Asimismo, podría mejorar al añadir ciertas relaciones entre las variables del modelo según el test de Lagrange, aunque no de una manera tan marcada. 


\section{MODELO 3: Replicación empírica de la dimensión Adaptabilidad}

Este modelo pretende verificar si los ítems 31 a 45 del cuestionario se agrupan en 3 factores: Orientación al cambio (ítems 31 a 35), Orientación al cliente (ítems 36 a 40) y Aprendizaje organizativo (ítems 41 a 45). Además se indicó que dichos factores (o subescalas) estaban correlacionados entre sí. $\mathrm{Al}$ analizar la media de los residuos estandarizados observamos que presenta valores relativamente bajos $(0,0434)$, de manera similar a lo que sucede con el error promedio de los elementos fuera de la diagonal $(0,0496)$. Por consiguiente, parece que el modelo presenta un ajuste aceptable, al menos en una primera aproximación. En lo que respecta a la distribución de los errores, éstos siguen una distribución más o menos simétrica y relativamente centrada en 0 (nótese en este punto que el intervalo entre 0,1 y $-0,1$ recoge en torno al $91,43 \%$ de los errores residuales). Por último, han sido necesarias 6 iteraciones para que el modelo pudiera converger. A partir de la quinta el cambio medio en las estimaciones de los parámetros del modelo no ha sufrido apenas modificaciones y se ha estabilizado en valores bajos.

Si atendemos a los diferentes índices de bondad de ajuste que se han calculado (Tabla 3) los resultados sugieren, aunque con algunas excepciones muy cercanas al nivel óptimo, que nos encontramos ante un modelo con un ajuste relativamente bueno, lo que parece por tanto corroborar la estructura interna de la adaptación del cuestionario por lo que se refiere a la dimensión Adaptabilidad y sus subescalas con sus respectivos ítems.

\section{MODELO 4: Replicación empírica de la dimensión Misión}

En el cuarto modelo se analizó si los 15 últimos ítems del cuestionario se agrupaban en 3 factores: Dirección y propósitos estratégicos (ítems 46 a 50), Metas y objetivos (ítems 51 a 55) y Visión (ítems 56 a 60). Del mismo modo que en todos los casos anteriores, se indicó que dichos factores estaban correlacionados entre sí. Dados los valores relativamente bajos de los residuos estandarizados o error promedio $(0,0291)$ y el error promedio de los elementos fuera de la diagonal $(0,0333)$, parece que el modelo en cuestión presenta un buen ajuste. En lo que respecta a la distribución de los errores, siguen una distribución más o menos simétrica y relativamente centrada en 0 (nótese en este punto que el intervalo entre 0,1 y $-0,1$ recoge en torno al 96,67\% de los mismos). Por último, si observamos las iteraciones que ha tenido que realizar el modelo hasta conseguir converger, han sido suficientes 5. Además, a partir de la tercera, el cambio medio en las estimaciones de los parámetros del modelo no ha sufrido apenas modificaciones y se ha estabilizado en valores bajos, lo que según Byrne (1994) puede significar que nos encontramos ante un ajuste adecuado. Así mismo, en prácticamente todos los índices de bondad de ajuste los valores son superiores a 0,90 y el SRMR es inferior a 0,05. En lo que respecta a los test de Wald y Lagrange el modelo no mejora eliminando ninguna de las relaciones planteadas. Todos estos datos sugieren que este modelo presenta un buen ajuste, incluso superior al primer modelo planteado para Implicación.

\section{MODELO 5: Replicación empírica de los factores de segundo orden}

En este modelo se introdujeron las puntuaciones medias en las 12 subescalas para contrastar si se agrupaban, como cabría esperar, en las 4 dimensiones culturales. Es decir, si Empowerment, Trabajo en equipo y Desarrollo de capacidades se unen a Implicación; si Valores centrales, Acuerdo y Coordinación e integración se asocian a Consistencia; si Orientación al cambio, Orientación al cliente y Aprendizaje organizativo se integran en Adaptabilidad; y si Dirección y Propósitos estratégicos, Metas y objetivos y Visión se agrupan con Misión. Además se propuso en el modelo que todas las dimensiones estaban relacionadas entre sí.

El modelo parece presentar un buen ajuste dados los valores bajos de los residuos estandarizados o error promedio $(0,0244)$ y el error promedio de los elementos fuera de la diagonal $(0,0288)$. Del mismo modo que en los modelos anteriores, por lo que respecta a la distribución de los errores, éstos 
siguen una distribución más o menos simétrica y relativamente centrada en 0 (nótese, de hecho, que el intervalo entre 0,1 y $-0,1$ recoge el $100 \%$ de los errores). Por último, si observamos las iteraciones que ha tenido que realizar el modelo hasta conseguir converger, han sido suficientes 5 . Además, a partir de la tercera, el cambio medio en las estimaciones de los parámetros del modelo no ha sufrido apenas modificaciones y se ha estabilizado en valores bajos. Los resultados de los diferentes índices de bondad de ajuste del modelo, ya que prácticamente todos son superiores a 0,90 y el SRMR inferior a 0,05 , también sugieren que e modelo es adecuado. En lo que respecta a los test de Wald y de Lagrange el modelo no mejora al eliminar ninguna de las relaciones planteadas entre las variables. En suma, la hipótesis de que las diferentes subescalas que conforman el cuestionario se agrupan en 4 dimensiones culturales o factores de segundo orden, es correcta para la adaptación española.

\section{Puntuaciones en la versión española del DOCS}

A continuación, en la Tabla 4, se resumen los principales estadísticos obtenidos de las diferentes dimensiones y subescalas que componen el cuestionario. Si comparamos los valores obtenidos en nuestro estudio con los alcanzados por Denison y colaboradores (ver sus trabajos de 2005 y 2006), se puede comprobar que ambos son muy parecidos, no observándose discrepancias relevantes. En general, los valores medios obtenidos en la muestra española suelen ser más elevados que en la muestra original, encontrándose la mayor diferencia en Empowerment y la menor en Orientación al cliente. La discrepancia promedio entre ambas muestras en cuanto a sus medias en las 12 subescalas es de 0,27 . En lo que respecta a la desviación típica, la mayor diferencia entre la muestra original y la adaptación española se encuentra en la escala Acuerdo, mientras que la menor se observa en Valores centrales. La desviación típica promedio entre las 12 subescalas es 0,017 .

Tabla 4: Mínimo, máximo, media y desviación típica (d.t.) de las dimensiones y subescalas que componen el DOCS en la versión española

\begin{tabular}{lccccc}
\hline DIMENSIÓN & N & Mínimo & Máximo & Media & D. T. \\
\hline IMPLICACIÓN & 487 & 1,47 & 5,00 & 3,67 &, 659 \\
CONSISTENCIA & 488 & 1,33 & 4,93 & 3,46 &, 605 \\
ADAPTABILIDAD & 485 & 1,07 & 5,00 & 3,43 &, 565 \\
MISIÓN & 485 & 1,20 & 5,00 & 3,45 &, 704 \\
\hline SUBESCALAS & & & & & \\
\hline Empowerment & 487 & 1,20 & 5,00 & 3,87 &, 704 \\
Trabajo en equipo & 487 & 1,00 & 5,00 & 3,51 &, 791 \\
Desarrollo de capacidades & 487 & 1,40 & 5,00 & 3,63 &, 713 \\
Valores centrales & 487 & 1,40 & 5,00 & 3,51 &, 669 \\
Acuerdo & 487 & 1,00 & 5,00 & 3,57 &, 734 \\
Coordinación e integración & 488 & 1,20 & 5,00 & 3,30 &, 695 \\
Orientación al cambio & 485 & 1,00 & 5,00 & 3,38 &, 672 \\
Orientación al cliente & 484 & 0,60 & 5,00 & 3,37 &, 667 \\
Aprendizaje organizativo & 485 & 1,00 & 5,00 & 3,53 &, 663 \\
Dirección y propósitos estratégicos & 485 & 1,00 & 5,00 & 3,48 &, 871 \\
Metas y Objetivos & 485 & 1,00 & 5,00 & 3,53 &, 771 \\
Visión & 485 & 1,00 & 5,00 & 3,35 &, 720 \\
\hline
\end{tabular}




\section{Discusión y conclusiones}

Los resultados obtenidos en el presente estudio, que deben ser corroborados en posteriores investigaciones mediante el análisis de nuevos datos, muestran una adecuada consistencia interna y validez de constructo para la adaptación al castellano del Denison Organizational Culture Survey (DOCS). La evidencia empírica presentada parece, en una primera aproximación, suficiente para avalar el uso de esta escala en su versión española. Aparecen, de todos modos, algunos aspectos que deben ser atendidos al utilizar esta versión, al tiempo que deberán ser mejorados en próximas adaptaciones de la misma.

De los 60 ítems analizados, todos funcionan bien excepto el ítem 19 que debería ser reformulado con el objetivo de aumentar su correspondencia con el resto de la escala. Creemos que es preferible esta opción antes que su eliminación porque, de este modo, se rompería la estructura interna que los autores originales siguieron en la construcción de esta encuesta. Salvo por el deficiente funcionamiento de este ítem (que ha afectado negativamente a la subescala Valores centrales), tanto las correlaciones entre las diferentes subescalas y dimensiones como la consistencia interna de las mismas han resultado sumamente adecuados y muy similares a los conseguidos por Denison, Janovics y Young (2005) y Denison, Janovics, Young y Cho (2006). Asimismo, de la comparación de medias se deduce que las puntuaciones de la versión inglesa y castellana de esta encuesta no presentan, en principio, discrepancias importantes.

Por otro lado, lo cual tiene una relevancia si cabe todavía mayor, los resultados observados en los diferentes AFC parecen justificar, al menos en parte, la estructura interna del DOCS en su adaptación al castellano. Algunas de las dimensiones y subescalas ofrecen mejor ajuste que otras, como es el caso de las dimensiones Implicación y Adaptación. La dimensión o rasgo cultural que obtiene menor respaldo empírico es Consistencia. Los valores para esta dimensión en los índices de bondad de ajuste así como en otros parámetros, no permiten concluir que nos encontremos ante un modelo con un buen ajuste. No obstante, dado que los resultados se encuentran próximos a lo que sería un modelo con un ajuste adecuado, tampoco parece oportuno desechar completamente dicha estructura. Habrá que continuar poniendo a prueba este modelo en próximas investigaciones. Por lo que hace referencia a la dimensión Adaptabilidad con sus subescalas, se puede afirmar que recibe cierto grado de confirmación pues cumple la mayoría de los requisitos establecidos. Finalmente, existe fuerte apoyo empírico en lo que concierne a la estructura de las 12 subescalas y su pertenencia a las 4 dimensiones culturales más generales.

Asimismo, como una limitación más a considerar, habrá que avanzar en el estudio de la validez convergente y discriminante del instrumento. Por otra parte, es conveniente ampliar la muestra objeto de estudio, ya que el hecho de encuestar solamente a personas vinculadas con el mundo de la investigación puede haber influido en los resultados hallados, al basarse éstos en una población relativamente homogénea.

En definitiva, el DOCS ha demostrado su utilidad tanto teórica como aplicada para evaluar los valores de la cultura organizacional que tienen una gran influencia a la hora de aumentar la efectividad que alcanzan las organizaciones (Denison, 1990, 2001; Denison, y Mishra, 1995; Gordon y DiTomaso, 1992; Kotter y Heskett, 1992). La adaptación al castellano que ahora se presenta de este instrumento, muestra suficiente apoyo empírico en base a los diferentes análisis realizados, lo que avala su utilización.

\section{Referencias}

Ashkanasy, N. M., Broadfoot, L. E. y Falkus, S. (2000). Questionnaire measures of organizational culture. En N. M. Ashkanasy, C. P. M. Wilderon y M. F. Peterson (Eds.), Handbook of Organizational Culture \& Climate (pp. 131-145). Thousand Oaks: Sage. 
Bonavia, T. y Quintanilla, I. (1996). La cultura de las organizaciones y sus efectos sobre la participación de los empleados. Revista de psicología del trabajo y de las organizaciones, 12 (1), 7-26.

Byrne, B. (1994). Structural Equation Modeling with EQS y EQS/Windows. Thousands Oaks: Sage.

Cook, J. D., Hepworth, S. J., Wall, T. D. y Warr, P. B. (1981). The Experience of Work. London: Academic Press.

Denison, D. R. (1990). Corporate Culture and Organizational Effectiveness. New York: John Wiley \& Sons.

Denison, D. R. (1996). What is the difference between organizational culture and organizational climate? A native's point of view on a decade of paradigm wars. Academy of Management Review, 21 (3), 619-654.

Denison, D. R. (2001). Organizational culture: can it be a key lever for driving organizational change? En C. L. Cooper, S. Cartwright y P. Ch. Earley (Eds.), The International Handbook of Organizational Culture and Climate (pp. 347-372). Chichester: John Wiley \& Sons.

Denison, D. R. y Mishra, A. (1995). Toward a theory of organizational culture and effectiveness. Organization Science, 6 (2), 204-223.

Denison, D. R. y Neale, W. (1994). Denison Organizacional Culture Survey. Ann Arbor: Aviat.

Denison, D. R. y Neale, W. (2000). Denison Organizational Culture Survey. Ann Arbor: Denison Consulting.

Denison, D. R. y Spreitzer, G. (1991). Organizational culture and organizational development: a competing-values approach. En R. W. Woodman y W. A. Passmore (Eds.), Research in Organizational Change and Development (pp 1-21). Greenwich: JAI Press.

Denison, D. R, Haaland, S. y Goelzer, P. (2003). Corporate culture and organizational effectiveness: is there a similar pattern around the world? Advances in Global Leadership, 3, 205-227.

Denison, D. R., Hart, S. L. y Kahn, J.A. (1996). From chimneys to cross-functional teams: developing and validating a diagnostic model. Academy of Management Journal, 39, 1005-1023.

Denison, D. R., Janovics, J. y Young, J. (2005). Diagnosing Organizational Cultures: Validating a Model and Method. Documento de trabajo. Denison Consulting Group.

Denison, D. R., Lief, C. y Ward, J. L. (2004). Culture in family-owned enterprises: recognizing and leveraging unique strengths. Family Business Review, 27 (1), 61-70.

Denison, D. R., Janovics, J., Young, J. y Cho, H. J. (2006). Diagnosing Organizational Cultures: Validating a Model and Method. Documento de trabajo. Denison Consulting Group.

Fey, C. y Denison, D. R. (2003). Organizational culture and effectiveness: can American theory be applied in Russia? Organization Science, 14 (6), 686-706.

Hambleton, R. K. (1994). Guidelines for adapting educational and psychological tests: a progress report. European Journal of Psychological Assessment, 10 (3), 229-244.

Hambleton, R. K. (1996). Adaptación de tests para su uso en diferentes idiomas y culturas: fuentes de error, posibles soluciones y directrices prácticas. En J. Muñiz (Coord.), Psicometría. Madrid: Universitas.

Kimberly, J. y Cook, J. M. (2008). Organizational measurement and the implementation of innovations in mental health services. Administration and Policy in Mental Health and Mental Health Services Research, 35 (1), 11-20.

Kotter, J. P. y Heskett, J. L. (1992). Corporate culture and performance. New York: Free Press. 
Maccallum, R. C.y Austin, J. T. (2000). Applications of structural equation modelling in psychological research. Annual Review of Psychology, 51, 201-226.

Marcone, R. y Martín, F. (2003). Construcción y validación de un inventario de cultura organizacional educativa (ICOE). Psicothema, 15 (2), 292-299.

Martínez García, N. (1994). Elaboración y validación de un instrumento para determinar el nivel de cultura organizacional en una empresa. Tesis Doctoral no publicada. UNAM, México D. F., México.

Muñiz, J. (1998). La medición de lo psicológico. Psicothema, 10 (1), 1-21.

Muñiz, J. y Hambleton, R. K. (2000). Adaptación de los tests de unas culturas a otras. Metodología de las Ciencias del Comportamiento, 2 (2), 129-149.

Price, J. L. y Mueller, C. W. (1986). Handbook of Organizational Measurement. Marshfield: Pitman Publishing Inc.

Reyes, A. M. y Zambrano, P. V. (1991). Un instrumento para medir la cultura organizacional innovadora. Tesis doctoral no publicada. Universidad Central, Santiago de Chile, Chile.

Rodríguez, C. M. (2005). Emergence of a third culture: shared leadership in international strategic alliances. International Marketing Review, 22 (1), 67-95.

Sánchez, J. C. y Alonso, E. (1997). La evaluación de la cultura: el cuestionario de conductas normativas organizacionales. Trabajo presentado al IV Congreso Nacional de Psicología Social. San Sebastián.

Schein, E. H. (1985). La cultura empresarial y el liderazgo. Barcelona: Plaza y Janés.

Scott, T., Mannion, R., Davies, H. y Marshall, M. (2003). The quantitative measurement of organizational culture in health care: a review of the available instruments. Health Services Research, 38 (3), 923-945.

Smerek, R. E. y Denison, D. R. (2007). Social capital in organizations: understanding the link to firm performance. En G. T. Solomon (Ed.), Proceedings of the Sixty-sixth Annual Meeting of the Academy of Management (CD).

Topa, G., Morales, J. F., Palací, F. (2005). El cumplimiento del contrato psicológico y su relación con la cultura organizacional para nuevos y veteranos: Un análisis multigrupo. Apuntes de Psicología, 23 (1), 53-70.

Van Muijen, J. J., Koopman, P., De Witte, K., De Cook, G., Susanj, Z., Lemoine, C., Bourantas, D., Papalexandris, N., Branyicski, I., Spaltro, E., Jesuino, J., Gonzalves das Neves, J., Pitaria, H., Konrad, E., Peiró, J., González-Romá, V. y Turnipseed, D. (1999). Organizational Culture: The Focus Questionnaire, European Journal of Work and Organizational Psychology, 8 (4), 551568. 
